\title{
Une initiative pédagogique intégrant les technologies d'information et de la communication (TIC) visant à rendre les sciences et l'informatique plus attrayantes pour les adolescentes
}

\section{A Pedagogical Initiative Integrating Information and Communication Technologies (ICT) with the Aim of Making Sciences and Computer Technology More Attractive to Girls Una iniciativa pedagógica que integra las tecnologías de la información y de la comunicación (TIC) que busca volver las ciencias y la informática más atractivas para las adolescentes}

\section{Nicole Lirette-Pitre et Donatille Mujawamariya}

Volume 33, numéro 1, printemps 2005

Les femmes en éducation et en formation

URI : https://id.erudit.org/iderudit/1079064ar

DOI : https://doi.org/10.7202/1079064ar

Aller au sommaire du numéro

\section{Éditeur(s)}

Association canadienne d'éducation de langue française

ISSN

0849-1089 (imprimé)

1916-8659 (numérique)

Découvrir la revue

Citer cet article

Lirette-Pitre, N. \& Mujawamariya, D. (2005). Une initiative pédagogique intégrant les technologies d'information et de la communication (TIC) visant à rendre les sciences et l'informatique plus attrayantes pour les adolescentes. Éducation et francophonie, 33(1), 89-104. https://doi.org/10.7202/1079064ar

\section{Résumé de l'article}

Cet article porte sur la problématique du manque d'intérêt et de confiance des filles dans les domaines scientifiques et technologiques à l'école comme sur le marché du travail. Dans leur tentative de contribuer à la résolution de ce problème, les auteures ont conçu des activités susceptibles d'intéresser les filles et de développer leur confiance en ces domaines. Les activités conçues s'inscrivent dans une perspective à la fois féministe et socioconstructiviste et utilisent les TIC. Les sujets retenus, pour des classes de sciences 9ième année du Nouveau Brunswick, bien que d'intérêt commun, ont été choisis de façon à aller chercher davantage les filles. De plus, ces activités ont été conçues de manière à ce qu'elles soient réalisées en équipes afin de favoriser la collaboration, la discussion, le dialogue, les échanges que sont les stratégies privilégiées par les filles dans leurs apprentissages. En recourant aux TIC, les auteures ont voulu permettre aux filles d'avoir des opportunités de s'exercer, d'apprivoiser les ordinateurs et de développer leur confiance en informatique.
Tous droits réservés (C) Association canadienne d'éducation de langue française, 2005 cest protége par la loi sur le droit d'auteur. L'utilisation des services d’Érudit (y compris la reproduction) est assujettie à sa politique d'utilisation que vous pouvez consulter en ligne. 


\section{Une initiative pédagogique intégrant les technologies d'information et de la communication (TIC) visant à rendre les sciences et l'informatique plus attrayantes pour les adolescentes}

\section{Nicole LIRETTE-PITRE}

Université de Moncton, Nouveau-Brunswick, Canada

\section{Donatille MUJAWAMARIYA}

Faculté des sciences de I'éducation, Université d'Ottawa, Canada

\section{RÉSUMÉ}

Cet article porte sur la problématique du manque d'intérêt et de confiance des filles dans les domaines scientifiques et technologiques à l'école comme sur le marché du travail. Dans leur tentative de contribuer à la résolution de ce problème, les auteures ont conçu des activités susceptibles d'intéresser les filles et de développer leur confiance en ces domaines. Les activités conçues s'inscrivent dans une perspective à la fois féministe et socioconstructiviste et utilisent les TIC. Les sujets retenus, pour des classes de sciences 9ième année du Nouveau Brunswick, bien que d'intérêt commun, ont été choisis de façon à aller chercher davantage les filles. De plus, ces activités ont été conçues de manière à ce qu'elles soient réalisées en équipes 
afin de favoriser la collaboration, la discussion, le dialogue, les échanges que sont les stratégies privilégiées par les filles dans leurs apprentissages. En recourant aux TIC, les auteures ont voulu permettre aux filles d'avoir des opportunités de s'exercer, d'apprivoiser les ordinateurs et de développer leur confiance en informatique.

\section{ABSTRACT}

\section{A Pedagogical Initiative Integrating Information and Communication Technologies (ICT) With the Aim of Making Sciences and Computer Technology More Attractive to Girls}

Nicole Lirette-Pitre

Faculty of Educational Sciences, Univeristy of Moncton, Canada

Donatille Mujawamariya

Faculty of Education, University of Ottawa, Canada

This article takes a look at the problem of girls' lack of interest and confidence for the scientific and technological fields at school and in the labour force. In their attempt to contribute to resolving this problem, the authors designed activities that could interest girls and develop their confidence in these fields. The activities are designed both from a feminist and socioconstructivist perspective, and use ICT. The topics for 9th grade New Brunswick science classes, although of common interest, were chosen to stimulate more interest among the girls. The activities were designed as group projects in order to promote collaboration, discussion, dialogue and exchange, strategies girls enjoy using when then are learning. By using ICT, the authors wanted to give girls opportunities to practise and get used to using computers, and to develop self-confidence in the field of computer technology.

\section{RESUMEN}

Una iniciativa pedagógica que integra las tecnologías de la información y de la comunicación (IIC) que busca volver las ciencias y la informática más atractivas para las adolescentes

Nicole Lirette-Pitre

Facultad de ciencias de la educación, Universidad de Moncton, Canadá

Donatille Mujawamariya

Facultad de educación, Universidad de Ottawa, Canadá

Este artículo aborda el problema de la falta de interés y de confianza de las muchachas en las áreas científicas y tecnológicas tanto en la escuela como en el mercado de trabajo. En su intento de contribuir a la solución de este problema, las autoras han concebido actividades susceptibles de interesar a las muchachas y desarrollar su 
confianza en esos campos. Las actividades concebidas se inscriben en una perspectiva a la vez femenina y socio-constructivista y utilizan las TIC. Los sujetos retenidos, para las clases de ciencias de 9 grado en Nuevo-Brunswick, aunque de interés común, se han escogido para que atraigan sobre todo a las muchachas. Además, estas actividades han sido concebidas para que se realicen en equipo con el fin de favorecer la colaboración, la discusión, el dialogo y los intercambios, que constituyen las estrategias privilegiadas por las muchachas en sus aprendizajes. $\mathrm{Al}$ recurrir a las TIC, las autoras han querido dar a las muchachas la oportunidad de ejercerse, de dominar las computadores y de desarrollar su confianza en informática.

\section{Introduction}

La réussite scolaire des filles les amène de plus en plus à poursuivre une formation post-secondaire (plus de $60 \%$ des baccalauréats en 2003 au Canada) et à exercer des professions demandant un haut niveau d'expertise. Les médias aidant, notre société a pris conscience de la féminisation de plusieurs professions telles que la médecine, l'enseignement, le droit, la psychologie, la sociologie, la biologie et la pharmacie (Stanton, 2003). Toutefois, cette situation en masque une très alarmante. Le fait que les filles sont très peu nombreuses à envisager une carrière scientifique ou technologique, soit une carrière de la nouvelle économie, parce qu'elles ignorent qu'elles ont les qualités essentielles pour y réussir (Lafortune et Solar, 2003; Gaudet et Lapointe, 2002). C'est pourquoi on retrouve peu de femmes en bioinformatique, en télémédecine, en ingénierie, en physique, et encore moins en informatique.

En 2000, de tous les diplômés universitaires en génie et en sciences appliquées au Canada, incluant les diplômés en informatique, seulement 24 pour cent étaient des femmes (Statistique Canada, 2003). Pour la même année, au Québec, les proportions d'étudiantes en génie et en physique ne dépassent pas $22 \%$ (Malavoy, 2003). Au Nouveau-Brunswick, les chiffres sont encore plus inquiétants. Une étude menée par Gaudet et Lapointe (2002) montre qu'en 1997, le taux d'inscription des jeunes femmes francophones à l'Université de Moncton en ingénierie, en informatique appliquée et en physique était respectivement de $17 \%, 15 \%$ et $6 \%$. La situation à cette université semble s'empirer davantage, car en 2003-2004, il n'y a eu aucune finissante en informatique appliquée ou en physique ${ }^{1}$. Mais comment expliquer ce manque d'intérêt et de confiance des filles à l'égard de ces disciplines?

Il semble que la plus grande perte d'intérêt et de la confiance en sciences et en informatique se manifeste au début du secondaire (Wigfield, Eccles et Pintrich, 1996; Acker et Oatley, 1993; AAUW, 1991). Pour plusieurs filles, le changement de l'école primaire à l'école secondaire a lieu au tout début de l'adolescence. Durant cette

1. Selon la liste des finissants 2003-2004 disponible à http://www2.umoncton.ca/cfdocs/finissants/finissants.cfm?order=Programme 
Notre objectif est de créer des apTIC qui intéressent davantage les adolescentes aux sciences et à l'informatique et qui augmentent, par leur utilisation en classe, leur confiance (auto-efficacité) en sciences et en informatique. période, plusieurs filles prennent conscience de leur rôle comme femmes et certaines sont très susceptibles aux stéréotypes. Par conséquent, leurs intérêts changent et elles ne se sentent pas du tout à l'aise dans les cours où les approches pédagogiques privilégient les intérêts et les habiletés des garçons, soient dans les cours de sciences et d'informatique (Papadimitriou, 2004; AAUW, 1991). Donc, l'école a un rôle important à jouer pour contrer cette perte d'intérêt et de confiance. En ce sens, plusieurs interventions québécoises dont entre autres le projet OPUS (Outils pédagogiques utiles en sciences) et des sites Internet comme Scientifines ont été mises en place (Deschênes, Sévigny, Foisy et Lemay, 2003; Théorêt et Garon, 2003). Toutefois, ces interventions ne sont pas très connues et sont peu utilisées au NouveauBrunswick. De plus, les curriculums de sciences de ces deux provinces, quoique semblables, ne sont pas identiques. Ce qui fait que l'utilisation du matériel développé au Québec n'est pas toujours pertinente dans les écoles du Nouveau-Brunswick.

Cet article porte sur la conceptualisation d'une recherche qui vise à concevoir des activités pédagogiques intégrant les technologies de l'information et de la communication (apTIC) pour le programme de sciences $9^{\mathrm{e}}$ dans des écoles francophones du Nouveau-Brunswick. Ces apTIC seront adaptées dans leur contenu, dans leur design et dans leurs approches pédagogiques tout en s'inspirant de la pédagogie féministe et de l'approche socioconstructiviste dans l'enseignement et l'apprentissage des sciences. Notre objectif est de créer des apTIC qui intéressent davantage les adolescentes aux sciences et à l'informatique et qui augmentent, par leur utilisation en classe, leur confiance (auto-efficacité) en sciences et en informatique. Dans la première partie de cet article, nous brossons un tableau succinct des facteurs affectant le choix de carrières des filles tout en nous attardant sur deux en particulier, l'intérêt et l'auto-efficacité. Par la suite, nous résumons les approches pédagogiques dont est inspirée notre étude et établissons le lien entre ces approches et les concepts d'intérêt et d'auto-efficacité. Nous discutons des éléments de design et du choix des sujets de sciences qui ont présidé à la conception des apTIC en question accompagnés d'un exemple d'illustration d'une apTIC. Nous concluons enfin sur l'impact présenti de ces apTIC relativement à l'intérêt et à la confiance des filles en sciences et en informatique.

\section{Facteurs qui affectent le choix de carrières des filles}

Il existe plusieurs modèles qui expliquent le choix de carrières des filles (Dickhäusser et Stiensmeier-Pelster, 2003; Nauta et Epperson, 2003; Eccles, 1994; Lent, Brown et Hackett, 1994). Ces modèles semblent s'accorder sur l'influence de l'intérêt et de la confiance en ses habiletés (l'auto-efficacité) dans un certain domaine (math, langues, etc.) sur le choix de poursuivre des études dans ce domaine (choix de cours au secondaire et ensuite à l'université) (Haines et Wallace, 2002; Diegelman et Subich, 2001; Tracey et Hopkins, 2001). Ces deux facteurs sont centraux dans la théorie sociocognitive du choix de carrière (Bandura, 2003; Hackett, 1995; Lent, et al., 1994). Comme le lecteur pourra le constater dans les pages qui suivent, 
LeUne initiative pédagogique intégrant les technologies d'information et de la communication (TIC)

visant à rendre les sciences et l'informatique plus attrayantes pour les adolescentes

l'intérêt et l'auto-efficacité sont des construits psychologiques qui font intervenir plusieurs autres facteurs.

\section{L'intérêt}

L'intérêt peut être divisé en intérêt individuel (intrinsèque) et intérêt situationnel (Baumert et Köller, 1996; Todt et Schreiber, 1996). L'intérêt individuel est une préférence personnelle pour une activité, un sujet, un sport, etc. Un intérêt individuel

Plusieurs filles n'ont pas d'intérêt (ou peu) en sciences et en informatique, car elles n'ont pas été placées dans un environnement éducationnel ou autre où elles pouvaient développer un intérêt dans ces domaines. fait partie du concept de soi d'une personne et, une fois développé, est relativement stable. Ce type d'intérêt se manifeste au fur et à mesure qu'un individu se développe. Un environnement riche d'expériences personnelles, de connaissances, d'opportunités, de défis élargit le champ d'intérêt d'un individu (Todt et Schreiber, 1996; Baker et Leary, 1995). L'intérêt individuel est un construit psychologique qui influence beaucoup la motivation intrinsèque d'une personne pour accomplir une certaine tâche ou pour atteindre un but ainsi que ses attitudes envers cette tâche et la valeur que la personne attribue à cette tâche. Cet intérêt est aussi influencé par les croyances d'une personne et par conséquent, est affecté par les stéréotypes sociaux. Plusieurs filles n'ont pas d'intérêt (ou peu) en sciences et en informatique, car elles n'ont pas été placées dans un environnement éducationnel ou autre où elles pouvaient développer un intérêt dans ces domaines (Britner et Pajares, 2001; Gardner, 1996). Très souvent cet environnement renvoie de messages subtils renforçant les stéréotypes sexistes qui contribuent à la reproduction des rapports sociaux inégaux (Mujawamariya et Guilbert, 2002). Ces auteures stipulent que :

... la société a choisi de valoriser les hommes plus que les femmes et a pour complices des hommes qui dans leur enseignement utilisent des stratégies qui ne permettent pas aux femmes de se démarquer et des outils d'évaluation inadaptés à la façon d'apprendre des femmes (p. 31).

Ce sont les intérêts individuels qui influencent les choix de cours aux secondaires et à l'université. Ce sont aussi ces intérêts qui sont déterminants dans le choix de carrières. Toutefois, ces intérêts peuvent changer suite aux expériences personnelles positives. Et qu'en est-il de l'intérêt situationnel?

L'intérêt situationnel est un intérêt provoqué par l'environnement, par une activité, par une tâche, par un sujet ou par un stimulus externe (Ainley, Hidi et Berndorff, 2002; Deci et Ryan, 2002; Todt et Schreiber, 1996). Les auteurs (Palmer, 2004; Hidi et Harackiewicz, 2000) affirment qu'une fois maintenu dans le temps, l'intérêt situationnel peut contribuer au développement de l'intérêt individuel. L'intérêt situationnel peut être engendré par un environnement éducatif qui en supporte le développement. Les recherches d'Hidi et Harackiewicz (2000) et celle de Palmer (2004) montrent que des sujets d'étude intéressants, des activités engageantes et authentiques et le travail de groupe sont tous des sources d'intérêt situationnel. Des chercheurs allemands ont eu recours à de telles sources pour intéresser les filles aux sciences physiques (Häussler et Hoffmann, 2002; Hoffmann, 2002). Ce qui a conduit à la modification du curriculum de physique dans les écoles secondaires de l'Allemagne. Pour notre part, nous nous inspirons de la pédagogie féministe et du 
socioconstructivisme pour créer des sources d'intérêt situationnel en sciences et en informatique pour les filles.

\section{L'auto-efficacité}

Selon Bandura (2003), l'auto-efficacité concerne les évaluations par l'individu de ses aptitudes personnelles. L'être humain a besoin d'une solide confiance en son efficacité pour commencer ou poursuivre l'effort requis afin de réussir une certaine tâche ou d'atteindre un but. L'auto-efficacité occupe une place centrale dans la théorie sociocognitive. En influençant le choix des activités et le degré de motivation, les croyances d'efficacité personnelle jouent un rôle important dans l'acquisition des compétences. Les croyances d'efficacité personnelle régulent également la motivation en façonnant les aspirations et les résultats attendus des efforts personnels (Schunk et Pajares, 2004; Bandura, 2003; Schunk et Pajares, 2002). Soulignons d'une part que l'auto-efficacité est spécifique dans la mesure où une personne peut se sentir compétente en langues et non en mathématiques. D'autre part, elle est contextuelle : une personne peut se sentir compétente en français, mais se sentir incapable d'écrire un roman (Schunk et Pajares, 2002). Bandura (2003) soutient qu'il existe quatre sources d'auto-efficacité : l'expérience active de maîtrise, l'expérience vicariante (comparaison sociale), la persuasion verbale et les états physiologiques et émotionnels. Pour les garçons, il semble que c'est surtout l'expérience active de maîtrise qui est la source la plus influente sur l'auto-efficacité (Bandura, 2003), alors que pour les filles, les quatre sources seraient également importantes (Zeldin et Pajares, 2000). De plus, les recherches (Bandura, 2003; Cooper et Weaver, 2003; Zeldin et Pajares, 2000) montrent que les garçons ont souvent tendance à surestimer leur auto-efficacité pour une tâche scientifique ou technologique (avec l'ordinateur), tandis que les filles sousestiment ou se sentent moins compétentes pour la même tâche même si elles ont un rendement équivalent à celui des garçons.

\section{Influence réciproque entre l'intérêt et l'auto-efficacité}

Selon la théorie sociocognitive, la croissance de l'intérêt individuel est stimulée par des réactions émotionnelles et d'efficacité personnelle. Un individu manifeste un intérêt durable pour des activités où il se sent efficace et qui lui procure de l'autosatisfaction. Il peut y avoir un décalage temporel entre des croyances d'efficacité récemment acquises et la croissance d'intérêt, donc parfois ça prend du temps à développer un intérêt individuel pour un domaine donné. Un sentiment élevé d'efficacité favorise des expériences de maîtrise qui, avec le temps, procurent de l'autosatisfaction conduisant à une augmentation de l'intérêt (Bandura, 2003; Tracey, 2002; Lent, et al., 1994).

Malheureusement, encore aujourd'hui, l'école et plus spécifiquement les classes de sciences et d'informatique, ne sont pas des environnements où les filles se sentent à l'aise de participer pleinement à leurs apprentissages et très souvent, les enseignants ignorent inconsciemment les besoins particuliers des filles, leurs intérêts et leurs processus d'apprentissage dans leur enseignement (Papadimitriou, 2004; Mujawamariya et Guilbert, 2002; Baker et Leary, 1995; Acker et Oatley, 1993; AAUW, 
La création des environnements éducatifs qui respectent les intérêts particuliers des filles et leurs façons d'apprendre pourraient être une intervention scolaire simple et efficace pour garder plus de filles en sciences et en informatique.
1991). Ce qui résulte en un manque d'intérêt dans le domaine scientifique et technologique chez les filles et un manque de confiance dans leurs capacités ainsi qu'en leurs habiletés. Ces expériences négatives risquent d'influencer leur choix de poursuivre les sciences et l'informatique comme domaines d'études. C'est pourquoi nous soutenons, comme d'autres auteurs d'ailleurs (Häussler et Hoffmann, 2002; Britner et Pajares, 2001), que la création des environnements éducatifs qui respectent les intérêts particuliers des filles et leurs façons d'apprendre pourraient être une intervention scolaire simple et efficace pour garder plus de filles en sciences et en informatique.

\section{Influence des approches pédagogiques d'inspiration féministe et socioconstructiviste sur l'intérêt et l'auto-efficacité}

Les discussions concernant l'adaptation des approches pédagogiques aux styles d'apprentissage des filles font appel à deux théories complémentaires. L'une s'inscrit dans le courant de pensée féministe et porte sur le mode d'apprentissage « intégré»². La deuxième découle de la psychologie cognitive et concerne l'approche socioconstructiviste en éducation. Nous avons choisi ces deux approches, car celles-ci semblent avoir une influence sur l'intérêt et sur l'auto-efficacité des filles en sciences et en informatique.

\section{Apprentissage intégré}

Une théorie sur les modes d'apprentissage (ou styles d'apprentissage) particulière aux femmes émerge des travaux réalisés par les psychologues américaines (Belenky, Clinchy, Goldberger et Tarule, 1986). Leur étude indique qu'indépendamment de l'âge, de la race ou du niveau scolaire, la majorité des femmes apprennent mieux dans un environnement où l'empathie est valorisée et où les expériences personnelles sont privilégiées. Un environnement éducationnel de ce genre permet à tous les apprenants, et surtout aux filles, de s'identifier aux autres et de mieux comprendre leurs points de vue, leurs idées, etc. L'apprentissage est plus significatif et important pour les filles si elles peuvent relier leurs idées et leurs expériences personnelles à celles des autres. Ce mode d'apprentissage est appelé "intégré». Contrairement au mode d'apprentissage "déconnecté» qui valorise la pensée critique, analytique et strictement objective ainsi que la compétition (Belenky, et al., 1986). Les personnes qui s'identifient au mode «intégré» se sentent plus à l'aise dans un environnement où elles peuvent collaborer et coopérer avec les autres. Elles utilisent leurs propres expériences de vie et leurs émotions pour relier le contenu du cours à leur vécu (Howes, 2002; Belenky, et al., 1986). .

2. Les termes mode d'apprentissage «déconnecté » et mode d'apprentissage «intégré » sont une traduction libre des termes "seperate knowing » et « connected knowing » utilisés par les psychologues américaines Belenky et al. (1986). 
Toutefois, les raisons psychologiques et biologiques de cette préférence restent encore inconnues. Cependant plusieurs chercheurs (Howes, 2002; Mayberry, 1999; Roychoudhury, Tippins et Nichols, 1995) présument que la socialisation et les rôles sociaux des hommes et des femmes pourraient être des facteurs importants pour expliquer ce phénomène. Quoi qu'il en soit, depuis longtemps, les approches pédagogiques prédominantes dans les classes de sciences sont l'enseignement magistral, le travail individuel, la mémorisation de faits, l'objectivation des savoirs, l'éloignement des savoirs scientifiques de l'activité humaine, etc. (Kennedy, 2004). Afin de rendre l'éducation scientifique plus équitable, il faudrait incorporer à l'enseignement des sciences des formes de pédagogie soucieuses de favoriser le mode d'apprentissage intégré (Howes, 2002).

En somme, si on veut créer des environnements pédagogiques intégrant les TIC qui stimulent le développement d'intérêts et augmentent l'auto-efficacité des filles, il convient de s'assurer que le contenu des cours de sciences soit relié le plus possible aux expériences personnelles des apprenantes, que leurs opinions et leurs idées personnelles soient valorisées, que la collaboration remplace la compétition, que le contexte soit riche et stimulant, que les sujets qui les intéressent soient traités en salle de classe et que la créativité et la pensée inductive soient valorisées (Lafortune et Solar, 2003; Mayberry, 1999; Roychoudhury, et al., 1995). D’où la nécessité de recourir à une approche socioconstructiviste.

\section{Le socioconstructivisme}

Le socioconstructivisme est une approche selon laquelle l'apprenant, à partir de ce qu'il sait déjà, construit peu à peu son savoir en interagissant avec les autres (enseignant et élèves) et son environnement (Howes, 2002). Une combinaison des approches féministes favorisant le mode d'apprentissage intégré et des approches socioconstructivistes contribue à la construction d'un environnement éducatif équitable pour tous les élèves (Howes, 2002; Roychoudhury, et al., 1995). Cette combinaison d'approches permet aux filles de relier leurs expériences personnelles à la matière enseignée et de se sentir valorisées pour leurs idées et leurs opinions. De plus, les filles travaillent en collaboration et dialoguent avec d'autres élèves (filles et garçons) afin de construire leurs connaissances (Kennedy, 2004). En collaboration, les élèves expriment leurs idées au sujet d'un concept, trouvent des arguments pour supporter leurs idées, discutent avec leurs collègues, dessinent ou schématisent leurs idées, etc. Cette approche engage les élèves dans la discussion et leur permet d'employer les concepts scientifiques et de construire une signification personnelle de ces concepts. Cette approche semble influer positivement sur les attitudes et sur la confiance des filles pour les sciences et pour la technologie (Mayberry, 1999).

Les activités pédagogiques conçues dans cette étude combinent les approches féministes favorisant le mode d'apprentissage intégré et l'approche socioconstructiviste. Les principes de l'approche socioconstructiviste et de la pédagogie féministe insistent notamment sur le travail de collaboration où les élèves dialoguent afin de construire leurs connaissances, sur l'apprentissage contextuel et authentique et sur la valorisation des expériences personnelles, des opinions et des idées. Les TIC peu- 
LeUne initiative pédagogique intégrant les technologies d'information et de la communication (TIC)

visant à rendre les sciences et l'informatique plus attrayantes pour les adolescentes

vent faciliter ce travail de collaboration et influer sur l'intérêt situationnel en sciences. De plus, un travail impliquant les TIC en sciences peut aussi influer positivement sur l'auto-efficacité en informatique (Oosterwegel, Littleton et Light, 2004; Todman et Drysdale, 2004; Cooper et Weaver, 2003; Hasan, 2003; Mayer-Smith, Pedretti et Woodrow, 2000).

\section{Apports des TIC aux approches féministe et socioconstructiviste}

Plusieurs chercheurs (Deschênes, et al., 2003; Lebrun, 2002; Mayer-Smith, et al., 2000) soulignent l'importance des TIC dans l'enseignement et l'apprentissage des sciences. En sciences, les TIC peuvent être utilisées comme outil pour faire la recherche d'informations et de données, le traitement de texte et d'images, le traitement de données brutes, des graphiques, des calculs, la simulation, etc. D'autres recherches (Deaudelin et Nault, 2003) montrent que les TIC facilitent beaucoup la communication lors d'un travail de collaboration. La communication constitue une part importante de l'activité scientifique, car elle intervient à toutes les étapes de la recherche scientifique (pour organiser l'investigation, pour partager les idées et les opinions, pour publier les résultats, etc.) Les TIC favorisent les interactions et les échanges qui contribuent essentiellement à la construction des connaissances par les élèves.

Lorsqu'on intègre des approches féministes favorisant le mode d’apprentissage intégré, ces activités deviennent des outils importants pour une éducation scientifique équitable et peuvent aider les filles à avoir des expériences positives et productives

en sciences et en informatique. et les experts est d'autant plus facile avec Internet, car ce réseau permet de réunir les participants, parfois dispersés géographiquement, autour d'un projet commun (Lebrun, 2002). Cette communication peut élargir le débat scientifique au-delà de la salle de classe et favoriser des échanges au sein d'une communauté d'élèves ou d'une communauté d'élèves et d'experts (Deaudelin et Nault, 2003). Ce dialogue, élément constitutif d'une construction sociale des connaissances, peut se faire à l'oral (partage des idées et des opinions, des informations, etc.), à l'écrit (sous forme de texte) ou à l'aide de média complémentaire (des images, des tableaux, des graphiques, des schémas, des animations, des vidéoclips, etc.) (Lebrun, 2002). Daudelin et Nault (2003) soulignent qu'un travail de collaboration qui intègre les TIC a plusieurs avantages. Il permet un partage et une mise en commun des idées et des points de vue (et les expériences personnelles) lors de la résolution de problèmes et il renforce la motivation des élèves et la perception de leur efficacité personnelle, car les élèves sont actifs et sont directement responsables de leurs apprentissage. Ainsi, dans une classe favorisant les approches socioconstructivistes, les TIC s'avèrent des outils très efficaces, voire essentiels.

Dans le cas d'apTIC conçues pour cette étude, la technologie ne constitue qu'un élément d'une configuration complexe d'apprentissage. Lorsqu'on intègre des approches féministes favorisant le mode d'apprentissage intégré, ces activités deviennent des outils importants pour une éducation scientifique équitable et peuvent aider les filles à avoir des expériences positives et productives en sciences et en informatique. Il convient de signaler que ces activités s'organisent autour d'un contenu (sujets d'intérêt des filles) qui constitue un autre facteur très important pour l'adaptation de l'enseignement des sciences et de l'informatique aux façons d'apprendre des filles. La présentation de ce contenu s'accompagne d'un support visuel dont le design doit 
également être pris en considération, car le design pourrait en soi créer un intérêt situationnel.

\section{Éléments retenus dans le design des apIIC et exemple d'une apIIC}

En plus des approches pédagogiques privilégiées dans les apTIC d'autres éléments du design sont susceptibles d'influencer l'intérêt situationnel des filles. Notons le contexte et les sujets traités dans l'apTIC, les couleurs, les images, les formes.

Les sujets scientifiques qui intéressent les filles diffèrent des sujets qui passionnent les garçons. Dans une recherche américaine Jones, Howe et Rua (2000) ont constaté que les filles aiment les sujets de science et de technologie en relation avec les êtres humains, les animaux, la société et l'environnement, et que le contexte est très important pour celles-ci (Howes, 2002). Notons que le contexte est l'ensemble des circonstances dans lesquelles s'insère un fait, un événement, une expérience, etc. En d'autres mots, le contexte est «l'histoire» qui accompagne l'activité. Aussi, les filles semblent plus intéressées aux sciences lorsqu'elles peuvent relier leurs expériences personnelles aux concepts étudiés (Mayberry, 1999).

Pour ce qui est du design visuel, il réfère aux images, aux couleurs, aux formes utilisées pour supporter l'enseignement. En effet, les images utilisées sont particulièrement importantes pour créer le contexte ou l'histoire de l'activité, elles sont très importantes pour les filles en favorisant l'établissement d'un lien entre les dimensions cognitives et affectives de l'apprentissage (Lynn, Raphael, Olefsky et Bachen, 2003; Jakobsdottir, Krey et Sales, 1994). Par conséquent, si le design de l'activité est attrayant pour les filles, elles auront plus tendance à vouloir explorer et faire l'activité en question.

Dans les apTIC conçues, nous avons pris le soin de tenir compte des couleurs et des formes préférées par les filles. En effet, ces apTIC contiennent une variété de couleurs pâles, des formes plutôt rondes et des images ou des photos de personnes, de plantes, ou d'animaux. De plus, chaque apTIC est contextuelle et authentique (touche la vie de tous les jours). La chimie des bijoux, les organismes modifiés génétiquement et notre alimentation, les voitures électriques, les produits secondaires de l'industrie spatiale sont entre autres les thèmes que nous avons retenus pour les apTIC développées. L’apTIC « La chimie des bijoux» est expliquée davantage dans la section suivante en guise d'illustration.

\section{Exemple d'une apTIC adaptée aux modes d'apprentissage des filles}

L'apTIC La chimie des bijoux (disponible dans le site web www.creatic.ca) est une activité reliée au programme de sciences naturelles de $9^{\mathrm{e}}$ année du Ministère de l'éducation du Nouveau-Brunswick. Celle-ci combine des approches pédagogiques intégrant le mode d'apprentissage intégré et le socioconstructiviste, de même que des éléments de contenu et de design préférés par les filles (couleurs pâles, formes plutôt rondes et images de personnes). 
La mise en situation de l'activité est la suivante: "Une compagnie de bijoux vient de s'installer au Nouveau-Brunswick. Cette compagnie offre un contrat à votre classe de sciences pour la construction de son site web. Ce site web portera sur la chimie des bijoux. Afin d'assurer que vous êtes les bons candidats et les bonnes candidates pour ce travail, la compagnie vous propose six petites tâches. Faites preuve de vos habiletés pour mériter ce contrat!». Les élèves travaillent en sous-groupes afin de compléter les six tâches directement à l'ordinateur. Ces tâches, sous forme de jeux-questionnaires, de jeux d'assemblage et de phrases à compléter sont faites directement dans Internet et portent sur les propriétés physiques et chimiques des métaux et des alliages, les formules chimiques et les structures cristallines. Une fois les tâches complétées, les groupes choisissent un sujet de recherche. Quelques exemples de sujets sont proposés : l'historique des bijoux, les bijoux de perçage, les bijoux et les allergies, les diamants au Canada, etc. En groupe, les élèves approfondissent leur sujet en faisant une recherche dans Internet et dans les livres. Par la suite, le groupe écrit un court texte multimédia comme synthèse de sa recherche. Après corrections par les pairs, les textes sont assemblés et le site Web est conçu. Tous les groupes ont une tâche spécifique dans la programmation du site et les groupes collaborent pour faire le design visuel du site. À tous les niveaux de l'activité, les élèves discutent, partagent leurs opinions, leurs idées, etc., afin d'employer les concepts scientifiques et techniques et de construire une signification personnelle de ces concepts. Afin d'aider l'enseignant dans sa planification et sa gestion de classe, l'apTIC est accompagnée d'un scénario pédagogique décrivant les cinq étapes de la démarche pédagogique 1) la mise en situation, 2) déroulement des activités à l'ordinateur, 3) l'objectivation, 4) l'évaluation et 5) le réinvestissement.

Dans cette apTIC, les approches féministes qui favorisent le mode d'apprentissage intégré sont privilégiées parce que :

- les élèves travaillent en collaboration tout au long de l'activité;

- l'enseignant fait une mise en situation qui permet aux filles (élèves) de relier leurs expériences personnelles au sujet d'étude;

- les élèves choisissent leurs sujets de recherche;

- les élèves partagent leurs idées, leurs opinions et leurs questions durant le travail et la présentation du site Web;

- la créativité est de mise tout au long de l'activité;

- l'objectivation permet de lier les nouvelles connaissances au vécu des élèves;

- le contenu de l'activité et le contexte valorisent le lien entre la science, les humains et la société.

Enfin, le socioconstructiviste est favorisé parce que :

- les élèves dialoguent afin de construire leurs connaissances;

- l'enseignant explore les connaissances antérieures des élèves (mise en situation);

- chaque membre de l'équipe a sa propre tâche à compléter (interdépendance) et participe à la recherche d'information, à la rédaction du texte et au développement du site Web;

- l'apprentissage est contextuel et authentique. 


\section{Conclusion}

En vue de promouvoir l'accès des filles aux études et aux carrières scientifiques et technologiques, nous avons conçu des apTIC susceptibles d'intéresser les filles et de développer leur confiance en ces domaines. Les activités conçues s'inscrivent dans une perspective à la fois féministe et socioconstructiviste et utilisent les TIC. Les sujets retenus, bien que d'intérêt commun, ont été choisis de façon à aller chercher

En vue de promouvoir l'accès des filles aux études et aux carrières scientifiques et technologiques, nous avons conçu des apTIC susceptibles d'intéresser les filles et de développer leur confiance en ces domaines. davantage les filles. De plus, ces activités ont été conçues de manière à ce qu'elles soient réalisées en équipes afin de favoriser la collaboration, la discussion, le dialogue, les échanges que sont les stratégies privilégiées par les filles dans leurs apprentissages. En recourant aux TIC, nous avons voulu permettre aux filles d'avoir des opportunités de s'exercer, d'apprivoiser les ordinateurs et de développer leur confiance en informatique.

Toutefois, comme l'indique le titre de notre texte, nous tenons encore une fois à souligner qu'il s'agit d'une initiative en voie d'exécution. Une fois toutes les apTIC conçues, nous avons entrepris des démarches qui nous permettront prochainement de mettre en œuvre ces activités auprès des élèves des classes de sciences $9^{\mathrm{e}}$ année du Nouveau Brunswick. Nous pourrons ainsi évaluer l'engouement des filles à ces activités et en même temps tenter d'expliquer leur intérêt et leur confiance à exécuter les tâches requises. Cette initiative en est également une de recherche-action dans la mesure où tout au long de la réalisation de notre étude, nous voulons inciter les enseignants titulaires des classes qui se seront portés volontaires à notre expérience de poursuivre l'utilisation de ces activités, de créer et d'implanter d'autres activités de même genre. À long terme, espérons-nous, l'initiative qui aura ses petits permettra de maintenir les filles en sciences et en technologie à l'école comme sur le marché du travail.

\section{Références bibliographiques}

AAUW. (1991). Shortchanging girls, shortchanging America. Washington, DC: American Association of University Women, 17 p.

ACKER, Sandra et OATLEY, Keith. (1993). Gender issues in education for science and technology: Current situation and prospects for change. Canadian Journal of Education, vol. 18, no 3, 255-272.

AINLEY, Mary, HIDI, Suzanne et BERNDORFF, Dagmar.(2002). Interest, learning, and the psychological processes that mediate their relationship. Journal of Educational Psychology, vol. 94, no 3, 545-561.

BAKER, Dale et LEARY, Rosemary. (1995). Letting girls speak out about science. Journal of research in science teaching, vol. 32, no 1, 3-27. 
BANDURA, Albert (2003). Auto-efficacité: Le sentiment d'efficacité personnelle. Bruxelles : De Boeck, 859 p.

BAUMERT, Jürgen et KÖLLER, Olaf (1996). Interest research in secondary I: An overview, dans Interests and learning. Proceedings of the Seon conference on interest and gender, sous la direction de Lore Hoffmann, Andreas Krapp, K. Ann Renninger et Jürgen Baumert. Kiel : IPN, 241-256.

BELENKY, Mary Field, CLINCHY, Blythe McVicker, GOLDBERGER, Nancy Rule et TARULE, Jill Mattuck (1986). Women's Ways of Knowing: The development of self, voice and mind. New York: Basic Books, 256 p.

BRITNER, Shari L. et PAJARES, Frank (2001). Self-efficacy beliefs, motivation, race, and gender in middle school science. Journal of Women and Minorities in Science and Engineering, vol. 7, 271-285.

COOPER, Joel et WEAVER, Kimberlee D. (2003). Gender and computers: Understanding the Digital Divide. New Jersey: Lawrence Erlbaum Associates, $168 \mathrm{p}$.

DEAUDELIN, Colette et NAULT, Thérèse (2003). Collaborer pour apprendre et faire apprendre: la place des outils technologiques. Québec: Presses de l'Université du Québec, 268 p.

DECI, Edward L. et RYAN, Richard M. (2002).Handbook of self-determination research. Rochester: University of Rochester Press.

DESCHENES, Claire, SÉVIGNY, Judith, FOISY, Martine et LEMAY, Anne-Marie (2003). Outils pédagogiques utiles en sciences (OPUS) : intéresser davantage les filles du secondaire aux sciences physiques, dans Femmes et maths, sciences et technos, sous la direction de Louise Lafortune et Claudie Solar. St-Foy, Québec: Presses de l’Université du Québec, 127-146.

DICKHÄUSSER, Oliver et STIENSMEIER-PELSTER, Joachim (2003) Gender differences in the choice of computer courses: applying an expectancy-value model. Social Psychology of Education, vol. 6, 173-189.

DIEGELMAN, Nathan M. et SUBICH, Linda Mezydlo (2001). Academic and vocational interests as a function of outcome expectancies in social cognitive career theory. Journal of Vocational Behavior, vol. 59, 394-405.

ECCLES, Jacquelynne S. (1994). Understanding women's educational and occupational choices. Psychology of Women Quarterly, vol. 18,585-609.

GARDNER, Paul.L. (1996). The development of males' and females' interests in science and technology, dans Interests and learning. Proceedings of the Seon conference on interest and gender, sous la direction de Lore Hoffmann, Andreas Krapp, K. Ann Renninger et Jürgen Baumert. Kiel : IPN, 41-57.

HACKETT, GaiL (1995). Self-efficacy in career choice and development, dans Self-efficacy in changing societies, sous la direction de Albert Bandura. New York, NY: Cambridge University Press, 232-258. 
HAINES, Valerie A. et WALLACE, Jean (2002). Exploring the association of sex and majoring in science. The Alberta Journal of Educational Research, vol. XLVIII, no 2, 188-192.

HASAN, B. (2003). Influence of specific computer experiences on computer self-efficacy beliefs. Computers in Human Behavior, vol. 19, no 4, 443-450.

HÄUSSLER, Peter et HOFFMAN, Lore (2002). An intervention study to enhance girls' interest, self-concept, and achievement in physics class. Journal of Research in Science Teaching, vol. 39, no 9, 870-888.

HOFFMANN, Lore (2002). Promoting girls' interest and achievement in physics classes for beginners. Learning and Instruction, vol. 12, 447-465.

HOWES, Elaine V. (2002). Connecting girls and science: Constructivism, feminism and science education reform. New York, NY: Teachers College Press, 167 p.

JAKOBSDOTTIR, Solveig, KREY, Cynthia L. et SALES, Gregory C. (1994). Computer graphics: Preferences by gender in grades 2, 4, and 6. Journal of Educational Research, vol. 88, no 2, 91-100.

JONES, Gail, HOWE, Ann et RUA, Melissa J. (2002). Gender differences in students' experiences, interests, and attitudes toward science and scientists. Science Education, vol. 84, 180-192.

KENNEDY, Margaret E. (2004). Meaning-making in the elementary classroom through collaboration. Rapport intérimaire. Ottawa: Université d'Ottawa, Faculté d'éducation, $138 \mathrm{p}$.

LAFORTUNE, Louise et SOLAR, ClaudiE (2003). Femmes et maths, sciences et technos. St-Foy, Québec : Presses de l'Université du Québec, 266 p.

LEBRUN, Marcel (2002). Des technologies pour enseigner et apprendre. Bruxelles: De Boeck Université, 240 p.

LEBRUN, Marcel (2002). Théories et méthodes pédagogiques pour enseigner et apprendre : quelle place pour les TIC dans l'éducation? Bruxelles : De Boeck Université, 206 p.

LENT, Robert W., BROWN, Steven D. et HACKETT, Gail (1994). Toward a unifying social cognitive theory of career and academic interest, choice, and performance. Journal of Vocational Behavior, vol. 45, no 1, 79-122.

LYNN, Kathleen-M., RAPHAEL, Chad, OLEFSKY, Karin et BACHEN, Christine M. (2003). Bridging the gender gap in computing: An integrative approach to content design for girls. Journal of educational computing research, vol. 28, no 2,143-162.

MALAVOY, Sophie (2003). La conquête inachevée. Gazette des femmes, vol. 25, no 6, 16-29. 
MAYBERRY, Maralee (1999). Reproductive and resistant pedagogies: The comparative roles of collaborative learning and feminist pedagogy in science education, dans Meeting the challenge: Innovative feminist pedagogies in action, sous la direction de Maralee Mayberry et Ellen Cronan Rose. New York, NY: Routledge, 1-22.

MAYER-SMITH, Jolie, PEDRETTI, Erminia et WOODROW, Janice (2000). Closing the gender gap in technology enriched science education: A case study. Computers \& Education, vol. 35, no 1, 51-63.

MUJAWAMARIYA, Donatille et GUILBERT, Louise (2002). L'enseignement des sciences dans une perspective constructiviste : vers l'établissement du rééquilibre des inégalités entre les sexes en sciences. Recherches féministes, vol. 15, no 2, 24-45.

NAUTA, Margaret M. et EPPERSON, Douglas L. (2003). A longitudinal examination of the social-cognitive model applied to high school girls' choices of nontraditional college majors and aspirations. Journal of Counseling Psychology, vol. 50, no 4, 448-457.

OOSTERWEGEL, Annerieke, LITTLETON, Karen et LIGHT, Paul (2004).Understanding computer-related attitudes through an idiographic analysis of gender- and self-representations. Learning and Instruction, vol. 14, 215-233.

PALMER, David (2004). Situational interest and the attitudes towards science of primary teacher education students. International Journal of Science Education, vol. 26, no 7, 895-908.

PAPADIMITRIOU, Michael (2004). What Girls Say About Their Science Education Experiences: Is Anybody Really Listening? : Trafford Publishing, $214 \mathrm{p}$.

ROYCHOUDHURY, Anita, TIPPINS, Debora J. et NICHOLS, Sharon E. (1995). Gender-inclusive science teaching: A feminist-constructivist approach. Journal of Research in Science Teaching, vol. 32, no 9, 897-924.

SCHUNK, D. et PAJARES, F. (2004). Self-efficacy in education revisited: Empirical and applied evidence, dans Research on sociocultural influences on motivation and learning, sous la direction de Greenwich : CT : Information Age Publishing, 115-138.

SCHUNK, Dale H. et PAJARES, Frank (2002). The development of academic self-efficacy, dans Development of achievement motivation, sous la direction de A. Wigfield et J. Eccles. San Diego : Academic Press.

STANTON, Danielle (2003). Féminisation des professions: Un problème? Gazette des femmes, vol. 25, no 2, 20-29.

STATISTIQUE CANADA (2003). Diplômés universitaires selon le domaine d'études et le sexe. Document télé-accessible à http://www.statcan.ca/francais/Pgdb/ educ21_f.htm (site consulté le 14 juin, 2003) 
THEORET, Manon et GARON, Roseline (2003). Les représentations des intervenantes des Scientifines sur le développement des femmes, la science et le pouvoir, dans Femmes et maths, sciences et technos, sous la direction de Louise Lafortune et Claudie Solar. St-Foy, Québec: Presses de l'Université du Québec, 225-245.

TODMAN, John et DRYSDALE, Emma (2004). Effects of qualitative differences in initial and subsequent computer experience on computer anxiety. Computers in Human Behavior, vol. 20, 581-590.

TODT, Eberhard et SCHREIBER, Susanne (1996). Development of interests, dans Interests and learning. Proceedings of the Seon conference on interest and gender, sous la direction de Lore Hoffmann, Andreas Krapp, K. Ann Renninger et Jürgen Baumert. Kiel : IPN, 25-40.

TRACEY, Terence J.G. (2002). Developement of interests and competency beliefs: A 1-year longitudinal study of fifth- to eighth-grade students using the ICA-R and structural equation modeling. Journal of Counseling Psychology, vol. 49, no 2,148-163.

TRACEY, Terence J.G. et HOPKINS, Nathaniel (2001). Correspondence of interests and abilities with occupational choice. Journal of Counseling Psychology, vol. 48, no 2, 178-189.

WIGFIELD, Allan, ECCLES, Jacquelynne et PINTRICH, Pau.l R. (1996). Development between the ages of 11 and 25, dans Handbook of educational psychology, sous la direction de David C. Berliner et Robert C. Calfee. New York, NY: Simon \& Schuster Macmillan, 148-185.

ZELDIN, Amy I. et PAJARES, Frank (2000). Against the odds: Self-efficacy beliefs of women in mathematical, scientific, and technological careers. American Educational Research Journal, vol. 37, no 1, 215-246. 